\title{
Atomistic simulation of ion irradiation of semiconductor heterostructures
}

\section{Fridlund, Christoffer}

2017-10-18

Fridlund , C , Laakso , J , Nordlund, K \& Djurabekova , F 2017 , ' Atomistic simulation of ion irradiation of semiconductor heterostructures ' , Nuclear Instruments \& Methods in Physics Research. Section B: Beam Interactions with Materials and Atoms , vol. 409 , pp. 14-18 . https://doi.org/10.1016/j.nin

http://hdl.handle.net/10138/300209

https://doi.org/10.1016/j.nimb.2017.04.034

cc_by_nc_nd

acceptedVersion

Downloaded from Helda, University of Helsinki institutional repository.

This is an electronic reprint of the original article.

This reprint may differ from the original in pagination and typographic detail.

Please cite the original version. 


\title{
Atomistic Simulation of Ion Irradiation of Semiconductor Heterostructures
}

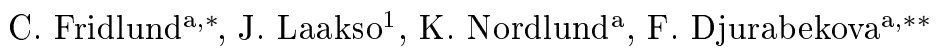 \\ ${ }^{a}$ Department of Physics and Helsinki Institute of Physics, University of Helsinki \\ P.O.Box 43 (Pietari Kalminkatu 2), Finland
}

\begin{abstract}
Recently the possibility to use ion beam mixing combined with suitable annealing has been suggested as a possible means to synthesize individual silicon quantum dots in a silica layer, with the possibility to function as single-electron transistors. For this to work, it is necessary to have a careful control of the ion beam mixing in $\mathrm{Si} / \mathrm{SiO}_{2} / \mathrm{Si}$ heterostructures, as well as understand the nature of not only the composition, but also the chemical modification of the $\mathrm{SiO}_{2}$ layer by the mixing with $\mathrm{Si}$. We describe here a procedure to synthesize $\mathrm{Si} / \mathrm{SiO}_{2} / \mathrm{Si}$ heterostructures in molecular dynamics, with an energy minimization scheme to create strong and stable interfaces. The created heterostructures are irradiated at energies and fluences matching corresponding experiments. The results show a considerable degree of interface mixing, as expected. They also show some densification of the silica layer due to recoil implantation, and formation of a considerable number of coordination defects. Due to the strong covalent bonding in silicon and silica, the densification is not fully elastically relaxed even in the presence of a nearby surface.
\end{abstract}

Keywords: semiconductor heterostructures, ion beam mixing, molecular dynamics

\section{Introduction}

With more hand-held devices like smartphones, tablets, cameras, etc, connecting to the internet-ofthings, power consumption is increasing exponentially. A search for more power-efficient components, which can facilitate the transition to a new era, is natural. The currently available transistors inside integrated semiconductor circuits will soon have reached their limitations [1]. Single electron transistors (SET) are by nature consuming less power [2], due to the limited current needed to send a signal from the emitter to the collector. However, the currently available SETs need cryogenic temperatures to work properly and, hence, are not very practical. A stable performance of an SET at room temperature will create a strong platform for reduction of power consumption by numerous electronic devices in simultaneous use.

The functional principle of the SET is based on formation of a quantum dot embedded in a dielectric matrix. Formation of Si nanocrystals embedded in amorphous silica structure is a straightforward way to

\footnotetext{
${ }^{*}$ Corresponding author

**Principal corresponding author
} 
achieve the suitable structure in a CMOS compatible manufacturing process.

Earlier it was shown by both experimental and computer simulations that quantum dots can be formed in a- $\mathrm{SiO}_{2}$ ) by annealing $\mathrm{Si}_{x} \mathrm{O}_{2-x}$. Such meta-stable mixture can be formed by ion mixing of a $\mathrm{Si} / \mathrm{a}-\mathrm{SiO}_{2}$ interface caused by low-energy ion beam irradiation $[3,4,5,6,7,8]$. Using kinetic Monte Carlo (kMC) $[5,9]$, the results of annealing process can be assessed quite closely, provided that the initial density profiles of different components in all layers of multi-layered structure are well known.

In this work, we present the molecular dynamic (MD) simulations of the initial irradiation process of $\mathrm{SiO}_{2} / \mathrm{Si}$ layered simulation cell, resulting in the density profiles, which can be used in subsequent kinetic Monte Carlo simulations. The results are discussed and the potential pitfalls for accelerated MD simulations are underlined.

\section{Development of simulation approach}

\subsection{Irradiation condition}

The ion fluence needed to achieve sufficient atomic mixing during irradiation is approximately $2.5 \times 10^{15}$ $\mathrm{cm}^{-2}$, and can be reached with about 10000 ions over the surface of simulation cells of 10 s nanometers. Simulation of such amount of ions within reasonable time is computationally very demanding using a conventional MD algorithm. Acceleration of the simulation runs by applying suitable approximations is required. All MD simulations in the current work are performed by using PARCAS [10, 11] MD code, which is routinely used to simulate ion cascades in the parallel mode on multiple computer cores. Yet, depending on the number of ions and the chosen interatomic potential, the time required for a single run series to 10000 ions amounts to many days of simulations, which is not affordable for systematic industrial process optimization. In the current work, we explore possible ways to reduce the computational costs of high fluence irradiation without affecting the final result dramatically.

As an initial attempt to simulate high fluence irradiation at small computational costs, we simulated the $\mathrm{Ne}$ and $\mathrm{Si}$ ion multiple impacts on the structure consisted of amorphous $\mathrm{SiO}_{2}\left(\mathrm{a}-\mathrm{SiO}_{2}\right)$ and crystalline $(\mathrm{c}-\mathrm{Si})$ slabs. These ions were selected to investigate two ways of modification of layered structure: either by using highly focused Ne ion irradiation, available from a Helium Ion Microscope (HIM) [12], or a regular broad Si ion beam.

In the current work, we focus on the details of accelerated MD simulations, which aim to enable the realistic evaluation of ion mixing process for industrially acceptable simulation times. Although MD simulations rely on the choice of interatomic potentials, multi-body interactions as well as the possibility to follow the dynamics of variation of atomic density due to collisional cascades and consequent buildup of stresses due to irradiation, make this type of simulations more attractive compared to the Binary Collision Approximation (BCA) approach [13]. To enable the detailed analysis of ion mixing in the studied structure, we simulate two cases of relatively small cells with two and one interfaces. While the size of the a- $\mathrm{SiO}_{2}$ cell is kept close 
to the experimental value of $7 \mathrm{~nm}$, the thickness of the c-Si slab was reduced for efficiency of the current simulations. The lateral size of all cells in this paper was $19.8 \mathrm{~nm}$. The interactions between the $\mathrm{Si}-\mathrm{Si}$ and $\mathrm{Si}-\mathrm{O}$ atoms were given by the Tersoff-like interatomic potential by Munetoh et al [14]. A schematic view of the regions of the structure can be seen in Fig. 1. To describe high-energy interactions, the ZBL potential [15] was joint to the Munetoh potential at short distances, and was also used to describe the interaction of $\mathrm{Ne}$ atoms with $\mathrm{Si}$ and $\mathrm{O}$ at all distances. More detail on how the irradiations were set up can be found in Sec. 2.3 .

In the following, we describe the construction of simulation cells and formation of a-SiO $2 /$ c-Si interfaces.

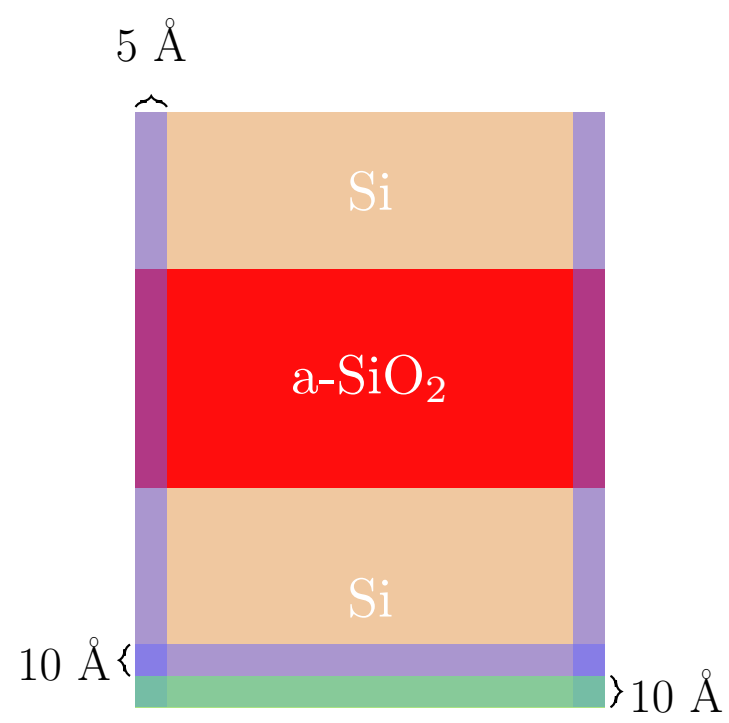

(a)

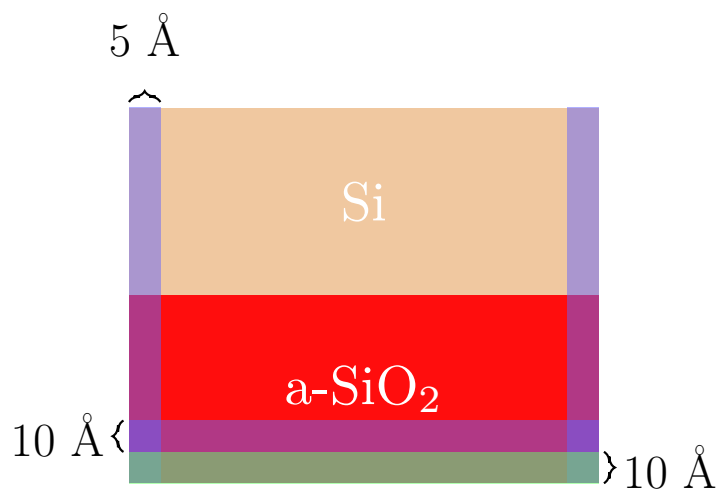

(b)

Figure 1: A schematic view of the simulation boxes with two a-SiO $\mathrm{S}_{2} / \mathrm{c}-\mathrm{Si}$ interfaces (a) and one a-SiO ${ }_{2} / \mathrm{c}-\mathrm{Si}$ interface (b) with corresponding sizes, which were used in the MD simulations of focused (a) and homogeneous (b) irradiation runs. The regions where the temperature was controlled to $300 \mathrm{~K}$ during irradiation runs are indicated in blue. The atoms fixed at the bottom to prevent the shift of the entire cell are shown in green.

\subsection{Preparation of heterostructures}

The simulation cell is a multilayered structure, consisting of an a- $\mathrm{SiO}_{2}$ layer embedded inside the c-Si matrix. The dimensions of the simulation cell were defined by experimental conditions, where the a-SiO $\mathrm{O}_{2}$ layer has a thickness of approximately $7 \mathrm{~nm}$. The lateral dimensions are chosen to enclose the entire cascade region to avoid the artefacts arising from periodic boundary conditions (PBC) and border temperature control on the sides of the cell. The stable a- $\mathrm{SiO}_{2}$ structure was obtained by relaxation of the initial $\mathrm{SiO}_{2}$ cell (obtained using the Wooten-Winer-Weaire method in Ref. [16]) and re-relaxed with Munetoh potential with PBC applied in all directions in the NPT ensemble at $300 \mathrm{~K}$, applying both Berendsen temperature and pressure controls with $\left(\tau_{T}=0.1 \mathrm{ps}\right.$ and $\left.\tau_{p}=0.1 \mathrm{ps}\right)$. The surrounding Si at the top and the bottom of the $\mathrm{a}_{-} \mathrm{SiO}_{2}$ was relaxed in crystalline form in such a way that the lateral dimensions of c-Si cell were 
perfectly matching the lateral dimensions of the $\mathrm{SiO}_{2}$ cell. This was important due to periodic boundaries to eliminate the concentration of artificial stresses on the sides of the cell affecting the results.

To create a multilayered structure, we merged the a- $\mathrm{SiO}_{2}$ and c-Si structures along the $z$-direction by compressing the c-Si matrix by $1.5 \AA$. Placing the compressed Si slabs at the top and the bottom of the a- $\mathrm{SiO}_{2}$ slab at different distances between the slabs, we could control the quality of the forming interface by monitoring the potential energy of the entire system (see Fig. 2). Periodic boundary conditions applied in all directions guaranteed that the stress due to compression relaxes towards the interface rather than at an open surface. The entire cell was allowed to relax for 250 ps. During this time, the Si parts sprung outwards towards their original size, filling the gap between the slabs and hence providing the fuse of the stacked structures. The separation distance $d$ of the slabs resulting in the lowest potential energy of the interface after relaxation was chosen as the most optimal structure (see Inset in Fig. 3, which shows that $d=1.2$ gave the minimal interface energy). In the case of a single interface, the structure was created in the similar manner as described here, however, the atoms around one of the interfaces were removed, and the structure was relaxed again with an open surface at the top and frozen layers at the bottom of the simulation cell. The dimensions of the structures used for irradiation simulations are shown in Fig. 1. In the figure, a)-c) images correspond to the focused irradiation and d)-f) are the homogeneous irradiation runs.

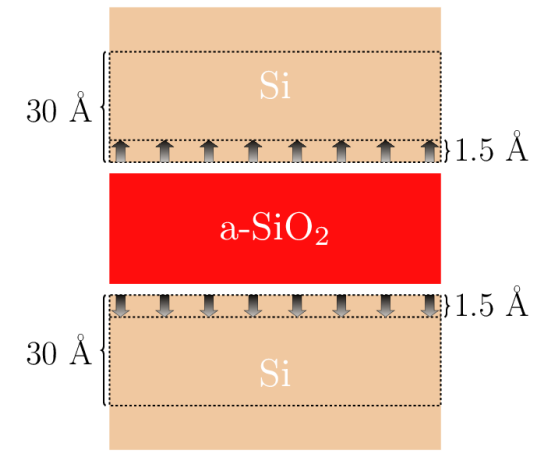

(a)

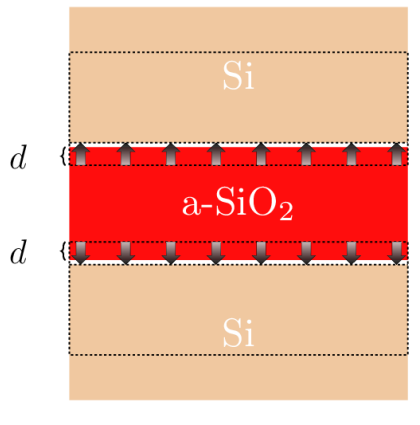

(b)

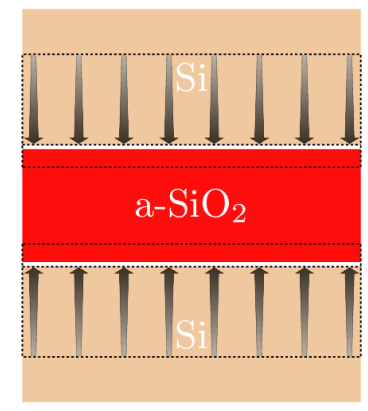

(c)

Figure 2: Illustration of how the slabs of $\mathrm{c}-\mathrm{Si}$ and $\mathrm{a}-\mathrm{SiO}_{2}$ were merged together. The thin outline is the guide for an eye to show the initial size of the c-Si slab. (a) Si structures are compressed by $1.5 \AA$ over a depth of $30 \AA$, (b) the Si distance $d$ between the compressed $\mathrm{Si}$ and $\mathrm{SiO}_{2}$ blocks is varied to achieve the optimal final interface, and (c) the new structure is relaxed to form strong bonds at the interfaces.

The heterostructure interface optimization method described here, is suitable for preparation of structures with multiple interfacing parts. During the relaxation, a huge shock-wave is generated, however, sufficiently long relaxation time $(\sim 250 \mathrm{ps})$ is able to suppress the oscillations. Furthermore, the method can easily be extended to any number of interfaces, provided that the relaxation time is adjusted accordingly. We made sure that the seams between the structures were stable, by minimizing the potential energy at the interface. 


\subsection{Irradiation procedure}

The first approximation adopted in the current simulations is reduction of relaxation runs after cascades. The irradiation process was run in as a series of consecutive irradiation simulations, and only every tenth was followed by a single relaxation run to reduce the build up stresses in previous cascades. As it was mention above (see Sec.2.1), two different types of simulation series were performed. In the runs with the focused $25 \mathrm{keV} \mathrm{Ne}^{+}$ion beams, we used a stacked structure with two interfaces (c-Si,3 nm - a-SiO${ }_{2}, 7 \mathrm{~nm}-$ c-Si,6 nm), see Fig. 1. For the broad $\mathrm{Si}$ beam, we used a homogeneous $3.7 \mathrm{keV} \mathrm{Si}{ }^{+}$ion irradiation of the structure with a single interface (c-Si,6 nm - a- $\left.\mathrm{SiO}_{2}, 3 \mathrm{~nm}\right)$.

The irradiation runs were performed in a quasi-NVE ensemble with temperature controlled by the Berendsen thermostat [17] only at the borders of the simulation cell within $5 \AA$ from the border. To precent a possible shift of the entire cell due to the momentum transferred from the incident ions, the three lowest layers of atoms were fixed. Above these three layers, the region of $10 \AA$ thick was also controlled to $300 \mathrm{~K}$ to avoid intensive interaction of energetic atoms with the frozen layers. Those atoms with energies sufficiently high to knock out lattice atoms were removed at the bottom of the cell, after they reached the region where the temperature was controlled.

As mentioned in Sec.2.1, we performed the simulations under two different irradiation condition: focused and broad ion beams. The focused irradiation simulation was tailored to correspond to an experimental samples, which comprised $25 \mathrm{~nm} \mathrm{Si}, 7 \mathrm{~nm} \mathrm{SiO}{ }_{2}$, and bulk Si with a thickness of at least $6 \mathrm{~nm}$ at the bottom, irradiated with $25 \mathrm{keV} \mathrm{Ne}^{+}$ions. To speed up the simulation of irradiation event even further, the cascades were initially simulated using the BCA code CASWIN [18] similarly to the approach used in [19]. The BCA simulations were run until the depth of $22 \mathrm{~nm}$, from where on, the simulations within the MD cell took over. After that, all energetic atoms including the incoming ions were transferred to the MD cell for subsequent MD simulations of the current cascade event. In these simulations we used periodic boundaries in all directions to avoid the direct sputtering too close to the interface.

We also simulated the broad ion beam condition. For this, we used lower ion energy, $3.7 \mathrm{keV}$, to enable the maximal ion mixing effect in the interface from the ions introduced at the open surface. We emulated the broad ion beam by a homogeneous distribution of ion impact positions. In practice, a new incoming ion was inserted at the lateral center of the structure, to initiate the cascades away from the border where the temperature was controlled. Before a subsequent ion, the simulation box was randomly shifted in the lateral directions.

Each cascade was allowed to develop for $500 \mathrm{fs}$ in the focused ion beam runs and $300 \mathrm{fs}$ in the homogeneous irradiation runs. After the ballistic phase of the cascade development, the temperature was rapidly quenched back to the room temperature $(300 \mathrm{~K})$ with the rate of $1 \mathrm{~K} / \mathrm{fs}$ to remove excess of kinetic energy from the cascade region. The duration of the ballistic phases of the cascades in both types of simulations was determined experimentally by running multiple test cases. After the quench, a short relaxation time for 
about $200 \mathrm{fs}$ was applied before the next ion is introduced into the system. After every 10 cascades, a more efficient relaxation run with the linear Berendsen temperature control with the target temperature of $300 \mathrm{~K}$ was performed for $5 \mathrm{ps}$.

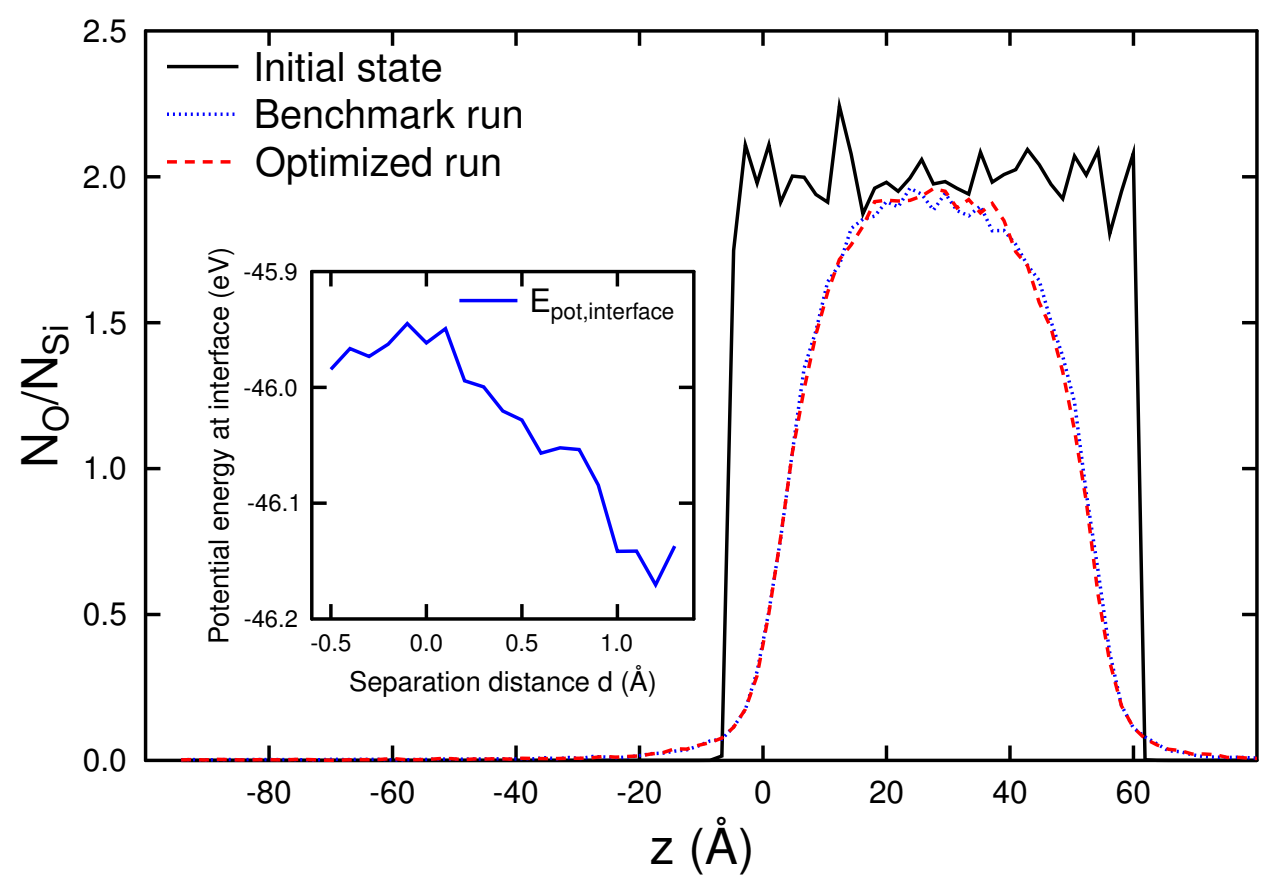

Figure 3: Comparison of Si profile broadening in a silica layer for two simulation runs, one with the speedup approaches, one without. The inset shows the interface potential with regards to separation distance, $d$. See Fig. 2 for the definition of $d$.

This procedure resulted in about 5 times faster calculation of the same fluence compared to the more conventional procedure. We also tested the reliability of the adopted speed-up scheme by performing an additional run of high fluence irradiation simulations for the larger cell with two interfaces and the recoils from the BCA simulations to emulate the focused He ion beam with the energy of $25 \mathrm{keV}$. The time between the irradiations was 5 ps, and each relaxation run was 2 ps. Comparison of the graphs in Fig. 3 shows that the profile broadening is practically identical with and without the speeding up scheme.

\section{Results and Discussion}

The simulations were run until 6500 ions were introduced in both focused and homogeneous simulations. This corresponds to the fluence of $1.6 \times 10^{15} \mathrm{~cm}^{-2}$ in the homogeneously irradiated cells. Fig. Fig. 4 shows the depth density profiles for different species ( $\mathrm{Si}$ and $\mathrm{O}$ ) in the simulation cell before and after 6500 ions were introduced in the cells. Additionally, we show the excess of Si atoms, which is calculated with respect to stoichiometric $\mathrm{Si}$ in a-SiO${ }_{2}$. Since initially there are no $\mathrm{O}$ atoms in $\mathrm{c}-\mathrm{Si}$, the $\mathrm{Si}$ atoms in this part are all considered to be "excess $\mathrm{Si}$ atoms". The figure exhibits very similar tendency of penetration of excess 
$\mathrm{Si}$ atoms into a-SiO $\mathrm{S}_{2}$ layer. Moreover, the picture does not depend on the presence of a second c-Si layer. However, we also observe a strong compression of the $\mathrm{a}_{-} \mathrm{SiO}_{2}$ layer, which also leads to the shift of the interface region deeper into the cell. This compression is explained by the momentum transfer from the ions to the atoms of the matrix and formation of many oxygen interstitials causing the increase of the $\mathrm{O}$ density in the $\mathrm{a}_{-} \mathrm{SiO}_{2}$ layer (see Fig. 4). However, while this process is rather natural, we believe that the effect may be exaggerated by the limited time scale of MD simulations.

Since the overall ration of Si:O still keeps intact as 1:2, we believe that quantitatively the results of ion mixing are reliable. We obtain a similar abundance of silicon to the one shown in [8]. At the same time, some reduction in atomic density of c-Si is also observed (from $\sim 0.05 \AA^{-3}$ to $\sim 0.04 \AA^{-3}$, in Fig. 4), which is related to the amorphization of the $\mathrm{c}-\mathrm{Si}$.

As a first step towards understanding the chemical nature of the material modification, we made statistics of the change of the coordination numbers in the homogeneous irradiation runs. In Fig. 5 we show the coordination of $\mathrm{Si}$ and $\mathrm{O}$ atoms separately in the initial cell and after irradiation with 6500 ions. In the initial structures, almost all Si atoms are 4-fold coordinated and $\mathrm{O}$ atoms 2-fold coordinates, as expected from the ideal chemical bonding. The small number of atoms with different coordination are due to interface defects. After the irradiation, a large fraction of both $\mathrm{Si}$ and $\mathrm{O}$ have a different coordination from the ideal one. Si has a roughly equal number of under- and over-coordinated atoms. This corresponds, at this high dose, to coordination defects in amorphous Si. This is well in line with recent experiments of the amorphization of Si which show that irradiated, unannealed Si does have a large fraction [20] of coordination defects.

The $\mathrm{O}$ atoms, on the other hand, do have a much larger fraction of overcoordinated than undercoordinated atoms. The $\mathrm{O}$ atoms with high coordination states correspond to atoms in interstitial positions, which clearly correlates with the observation of a high-density region in the silica layer after irradiation (Fig. 4).

\section{Conclusions}

We performed accelerated molecular dynamics simulations of high fluence irradiation of $\mathrm{Si}-\mathrm{SiO} 2$ heterostructures with the aim of increasing the computational efficiency. Such approach was motivated by the necessity of fast estimation of concentration of over-stoichiometric $\mathrm{Si}$ atoms in a-SiO2 layer to enable the formation of isolated Si nanocrystals, which can be used in manufacturing of single electron transistors. We showed that, by reducing the relaxation time between cascade runs, the 5 times speed-up of the simulations can be achieved. We also shodisplaygrawed that we obtain reasonable profiles with somewhat densified a-SiO${ }_{2}$ layer caused by $\mathrm{O}$ interstitials generated in the cascades. However, the density profiles compare well with the previous observations and the analysis of the coordination number before and after irradiation shows reasonable stoichiometry of $\mathrm{SiO}_{2}$ with the tendency of increasing the number of over-coordinated $\mathrm{O}$ atoms in $\mathrm{SiO}_{2}$ layer after high fluence irradiation. 


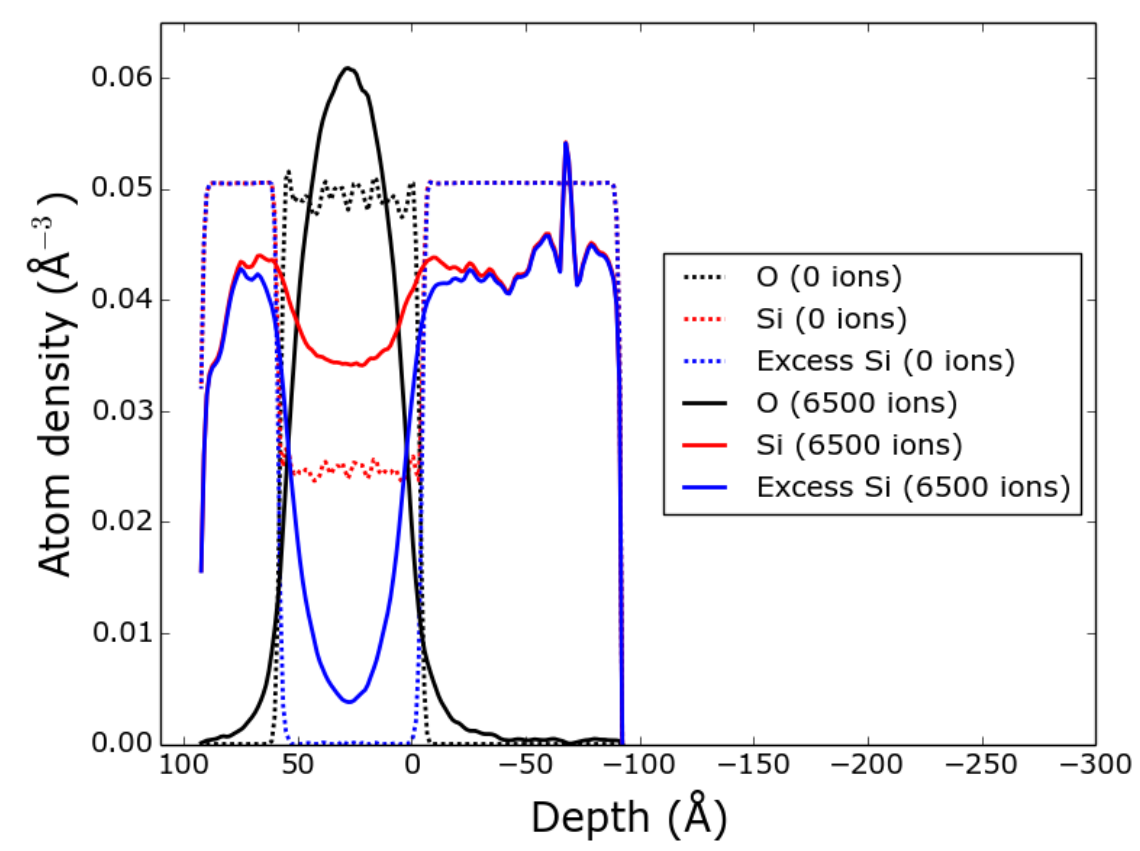

(a)

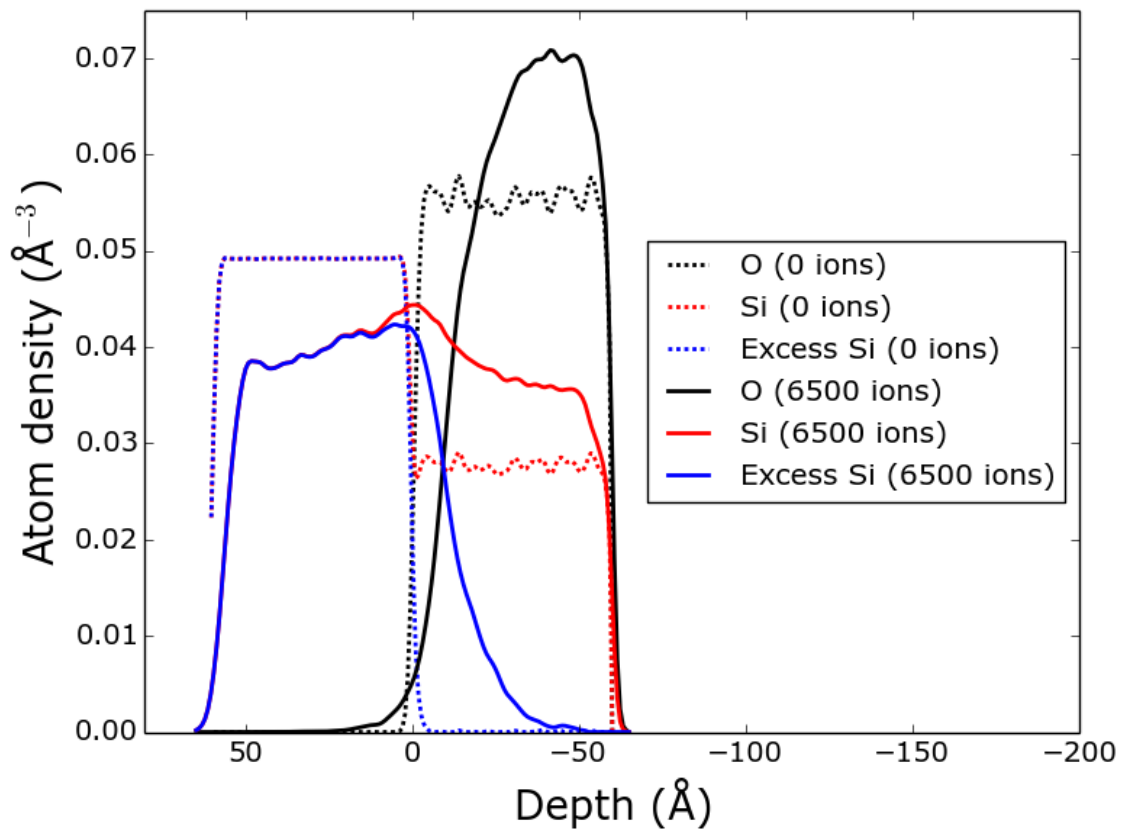

(b)

Figure 4: The density profiles of the (a) focused irradiation and (b) the heterogeneous irradiation. The dashed lines indicates the densities before the irradiation, and the solid lines indicates the state of the system after 6500 ions. In both (a) and (b) is the top surface of the system to the left. "Excess $\mathrm{Si}$ " is defined as the fraction of silicon exceeding the stoichiometric composition $\mathrm{Si}_{1} \mathrm{O}_{2}$, calculated as $N_{\text {exceessSi }}=N_{\mathrm{Si}}-N_{\mathrm{O}} / 2$ layer by layer (since there is no oxygen in the pure Si part, the excess $\mathrm{Si}$ number seems large there). 


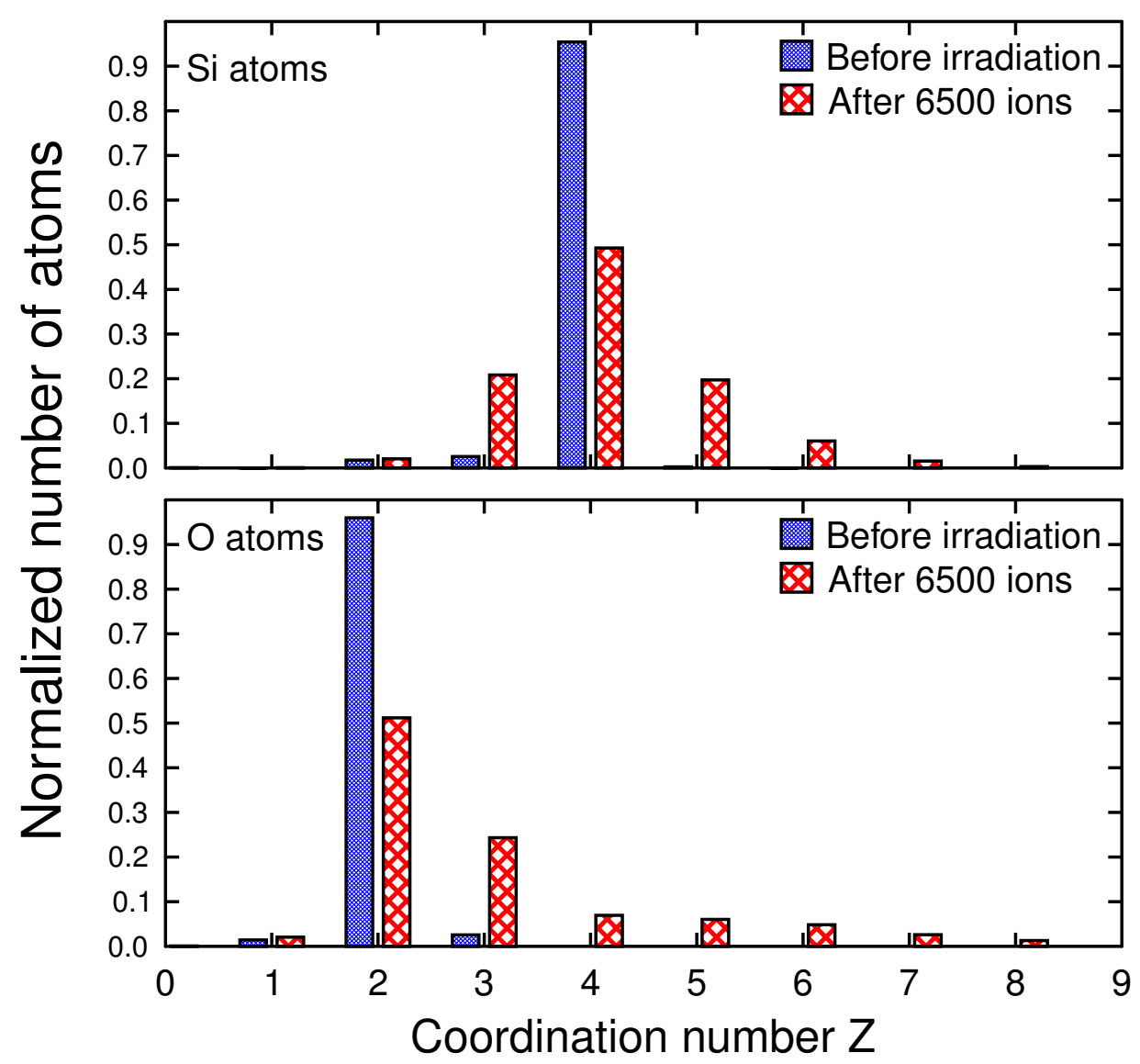

Figure 5: Distribution of coordination numbers of Si and O atoms before and after 6500 ions.

\section{Acknowledgements}

This work has been funded by the European Union's Horizon 2020 research and innovation program under grant agreement No 688072 .

[1] D. L. Feldheim, C. D. Keating, Self-assembly of single electron transistors and related devices, Chem. Soc. Rev. 27 (1) (1998) 1. doi:10.1039/a827001z.

[2] S. Lee, Y. Lee, E. B. Song, T. Hiramoto, Observation of Single Electron Transport via Multiple Quantum States of a Silicon Quantum Dot at Room Temperature, Nano Lett. 14 (1) (2014) 71-77. doi:10.1021/nl403204k.

[3] P. Normand, K. Beltsios, E. Kapetanakis, D. Tsoukalas, T. Travlos, J. Stoemenos, J. Van Den Berg, S. Zhang, C. Vieu, H. Launois, J. Gautier, F. Jourdan, L. Palun, Formation of 2-D arrays of semiconductor nanocrystals or semiconductorrich nanolayers by very low-energy Si or Ge ion implantation in silicon oxide films, Nucl. Instruments Methods Phys. Res. Sect. B Beam Interact. with Mater. Atoms 178 (1-4) (2001) 74-77. doi:10.1016/S0168-583X(01)00495-5.

[4] P. Novikov, K.-H. Heinig, A. Larsen, A. Dvurechenskii, Simulation of ion-irradiation stimulated Ge nanocluster formation in gate oxides containing GeO2, Nucl. Instruments Methods Phys. Res. Sect. B Beam Interact. with Mater. Atoms 191 (1-4) (2002) 462-467. doi:10.1016/S0168-583X(02)00593-1.

[5] T. Müller, K.-H. Heinig, W. Möller, Size and location control of Si nanocrystals at ion beam synthesis in thin SiO2 films, Appl. Phys. Lett. 81 (16) (2002) 3049. doi:10.1063/1.1512952.

[6] T. Müller, K.-H. Heinig, W. Möller, Nanocrystal formation in Si implanted thin SiO2 layers under the influence of an absorbing interface, Mater. Sci. Eng. B 101 (1-3) (2003) 49-54. doi:10.1016/S0921-5107(02)00711-0.

[7] L. Röntzsch, K.-H. Heinig, B. Schmidt, Experimental evidence of Si nanocluster $\delta$-layer formation in buried and thin SiO2 films induced by ion irradiation, Mater. Sci. Semicond. Process. 7 (4-6) (2004) 357-362. doi:10.1016/j.mssp.2004.09.098. 
[8] L. Röntzsch, K.-H. Heinig, B. Schmidt, A. Mücklich, W. Möller, J. Thomas, T. Gemming, Direct evidence of selfaligned Si nanocrystals formed by ion irradiation of Si/SiO 2 interfaces, Phys. status solidi 202 (15) (2005) R170-R172. doi:10.1002/pssa.200521399.

[9] E. A. Mikhantiev, I. G. Neizvestny, S. V. Usenkov, N. L. Shwartz, Silicon monoxide role in silicon nanocluster formation during Si-rich oxide layer annealing - Monte Carlo simulation, Comput. Mater. Sci. 90 (2014) 99-105. doi:10.1016/j.commatsci.2014.04.003.

[10] K. Nordlund, M. Ghaly, R. S. Averback, M. Caturla, T. Diaz de la Rubia, J. Tarus, Defect production in collision cascades in elemental semiconductors and fcc metals, Phys. Rev. B 57 (13) (1998) 7556-7570. doi:10.1103/PhysRevB.57.7556.

[11] K. Nordlund, J. Keinonen, M. Ghaly, R. S. Averback, Coherent displacement of atoms during ion irradiation, Nature 398 (6722) (1999) 49-51. doi:10.1038/17983.

[12] Ions4set Project, http://www.ions4set.eu/.

[13] M. T. Robinson, I. M. Torrens, Computer simulation of atomic-displacement cascades in solids in the binary-collision approximation, Phys. Rev. B 9 (12) (1974) 5008

[14] S. Munetoh, T. Motooka, K. Moriguchi, A. Shintani, Interatomic potential for Si-O systems using Tersoff parameterization, Comput. Mater. Sci. 39 (2) (2007) 334-339. doi:10.1016/j.commatsci.2006.06.010.

[15] J. F. Ziegler, J. P. Biersack, U. Littmark, The Stopping and Range of Ions in Solids, 1985.

[16] F. Djurabekova, K. Nordlund, Atomistic simulation of the interface structure of si nanocrystals embedded into amorphous silica, Phys. Rev. B 77 (2008) 115325, also selected to Virtual Journal of Nanoscale Science \& Technology Vol. 17 Issue 13 (2008).

[17] H. J. C. Berendsen, J. P. M. Postma, W. F. van Gunsteren, A. DiNola, J. R. Haak, Molecular dynamics with coupling to an external bath, J. Chem. Phys. 81 (8) (1984) 3684-3690. doi:10.1063/1.448118.

[18] T. S. Pugacheva, F. G. Djurabekova, S. K. Valiev, Effects of cascade mixing, sputtering and diffusion by high dose light ion irradiation of boron nitride, Nucl. Instruments Methods Phys. Res. Sect. B Beam Interact. with Mater. Atoms 141 (1-4) (1998) 99-104. doi:10.1016/S0168-583X(98)00139-6.

[19] M. Backman, F. Djurabekova, O. H. Pakarinen, K. Nordlund, Y. Zhang, M. Toulemonde, W. J. Weber, Cooperative effect of electronic and nuclear stopping on ion irradiation damage in silica, J. Phys. D. Appl. Phys. 45 (50) (2012) 505305. doi:10.1088/0022-3727/45/50/505305.

[20] E. Holmström, B. Haberl, O. Pakarinen, K. Nordlund, F. Djurabekova, R. Arenal, J. S. Williams, J. E. Bradby, T. C. Petersen, A. C. Y. Liu, Diversity in short-to-intermediate range order in pure forms of amorphous silicon generated by a variety of experimental and modeling techniques, J. Non-Cryst. Solids 438 (2016) 26. 